\title{
Comparative study between (Dexamethasone - levobupivacaine) versus (Neostigmine - levobupivacaine) for postoperative analgesia in Patient Undergoing Perianal Surgeries
}

\author{
Ahmed Hamody Hassan *; Wael Alham Mahmoud **;Khald abdelfattah Mohamed \\ Abdelfatah $* * *$ and Ahmed Abdelkahaar Aldardeer****
}

\begin{abstract}
Aim: This work aims to compare the postoperative analgesic effects and side effects of Dexamethasone or Neostigmine that added to levobupivacaine. Patient and Methods: Eighty patients aged between 20-80 years with ASA I- II who were scheduled for perianal surgeries under spinal anesthesia were enrolled in our study after written informed consent and approval of ethical committee; This Study was conducted in Sohag University Hospitals from August 2016 to March 2017. Patients Were divided into 2 equal groups:- (Group A) 40 patients received $100 \mu \mathrm{g}$ Neostigmine in $0.5 \mathrm{ml}$ normal saline added to $2.5 \mathrm{ml}$ volume of levobupivacaine.- (Group B) 40 patients received $4 \mathrm{mg}$ dexamethasone added to $2.5 \mathrm{ml}$ volume of levobupivacaine. Under complete aseptic conditions, spinal anesthesia was carried out in the sitting position, at level (L3-4 or L4-5). After a free flow of cerebrospinal fluid was confirmed, each patient received one of the coded spinal solutions (GA or GB). After administration; the patients stilled in sitting potion for 10 minutes then patients were turned into the supine position. Patients were monitored for: Heart rate; NIBP and Oxygen Saturation. Patients were observed for onset, duration of sensory block and motor block; In the post-anesthesia care unit (PACU), the patients were asked to assess their level of pain based on a visual analog scale (VAS). Complications also were observed .
\end{abstract}

Results: Dexamethasone had a faster Onset of sensory block onset than Neostigmine; Dexamethasone had a longer duration of regard Duration of motor block than Neostigmine; Neostigmine group had a longer analgesic effect stayed longer than Dexamethasone group .No Significant difference Between Two groups in rate of Complications ; Hypotension and Bradycardia were the most frequent complications in the two groups .Conclusion: the addition of Dexamethasone $4 \mathrm{mg}$ Neostigmine $100 \mu \mathrm{g}$ to intrathecally injected Levobupivacaine improved the effect of Levobupivacaine and the postoperative analgesic effect and duration

* Lecturer of anesthesia \& ICU **Lecturer of anesthesia \& ICU *** Lecturer of anesthesia \& ICU **** Lecturer of General surgery 


\section{Introduction}

-Spinal Anesthesia is the most commonly used technique for lower abdominal surgeries as it is very economical and easy to administer. (1).

-Spinal anesthesia has the advantage that profound nerve block can be produced in a large part of the body by the relatively simple injection of a small amount of local anesthetic (2).

-Spinal anesthesia avoids the risks of general anesthesia such as aspiration of gastric contents and difficulty with airway management. (1).

-However, postoperative pain control is a major problem because spinal anesthesia using only local anesthetics associated with relatively short duration of action, and thus early analgesic intervention is needed in the postoperative period ( 3 ). .

An increasing number of studies compare spinal levobupivacaine, an $\mathrm{S}$ (-)-enantiomer of bupivacaine, with racemic bupivacaine or other local anesthetics, either isobaric or hyperbaric, for obstetrics, orthopedics, herniorrhaphy, and transurethral surgeries (3)

-To improve the quality of central neuraxial block or spinal anesthesia, adjuvant drugs are used with local anesthetics. These drugs enhance or potentiate the actions of local anesthetics in order to improve the latency of the block, duration, and quality of analgesia. (4).

-Opioids that are added intrathecally prolong the duration of anesthesia, but are associated with a number of undesirable side e ects like delayed respiratory depression, urinary retention, pruritis, nausea, and vomiting. . (1).

-Drugs like dexmedetomidine, dexamethasone and magnesium sulfate have also been used successfully as an adjunct in spinal anesthesia along with local anesthetic (5).

-It is known that Dexamethasone has anti-inflammatory and analgesic action by inhibition of transmission in nociceptive C-fibers and neural discharge. When given as an additive in peripheral nerve blocks or in intrathecal anesthesia, it prolongs the duration of anesthesia. (1).

Reports indicate that IT cholinergic receptor agonists or cholinesterase inhibitors may modulate pain in animal and human studies. (4)

Intrathecal neostigmine produces analgesia but it also produces side effects, such as nausea and vomiting which could limit its use in clinical practice(4)

\section{Patients and Methods}

Eighty patients aged between 20-80 years with ASA I- II who were scheduled for perianal surgeries under spinal anesthesia were enrolled in our study after written informed consent and approval of ethical committee. This Study was conducted in Sohag University Hospitals from August 2016 to March 2017.Patientswere excluded if they had a history of Opium addiction, sedative drugs consumption; Contraindication for spinal anesthesia, as sepsis, severe coagulopathy and severe heart disease or Patient unable to communicate making postoperative assessment di cult.

Patients were divided into 2 equal groups:

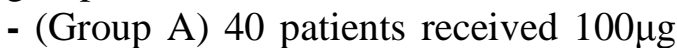
neostigmine in $0.5 \mathrm{ml}$ Normal saline added to $2.5 \mathrm{ml}$ volume of levobupivacaine

- (Group B) 40 patients received 4mg dexamethasone to $2.5 \mathrm{ml}$ volume of levobupivacaine. 
Then we did the followings:

1-A venous access was secured using wide bore cannula and the patient was preloaded with lactated ringer solution $(10 \mathrm{ml} / \mathrm{kg})$ before the induction of the spinal anesthesia

2- Under complete aseptic conditions, spinal anesthesia was carried out in the sitting position, at level (L2-3 or L3-4). After a free flow of cerebrospinal fluid was confirmed, each patient received one of the coded spinal solutions (GA or GB).

3- After administration, the patients were still in siting position for about 10 minutes (Saddle block) then patients were turned for into the supine position

4- Patients were monitored by:_Heart rate using ECG electrodes; NIBP Using NIBP Cable and Oxygen Saturation Using Pulse Oximetery. And baseline values were recorded.

5- Observations:

A-Patients were observed for onset, duration of sensory block bilaterally by using to pin prick with a short hypodermic needle

B-Motor blockade is assessed based on a modified Bromage scale:

Grade 0 no paralysis, able to flex extended leg at hip;

Grade 1 able to flex knee but not flex extended leg;

Grade 2 able to move foot only;

Grade 3 unable to move foot (Biswas et al; 2002)

These tests were performed every $5 \mathrm{~min}$ after the spinal block intra-operative and every 15 minutes postoperative for 3 hours until the sensory and motor variables were back to normal.

C-Intra operatively, the patients received $10 \mathrm{~mL} / \mathrm{Kg}$ lactated Ringer solution. Systolic ;Diastolic Blood Pressure and
Heart rate were recorded before spinal anesthesia and

there after every $5 \mathrm{~min}$ until the end of the procedure and every 15 minutes for 3 hours

D-Oxygen Saturation were recorded before spinal anesthesia and there after every $5 \mathrm{~min}$ until the end of the procedure

E-In the post-anesthesia care unit (PACU), the patients were asked to assess their level of pain based on a visual analog scale (VAS) ranging from 0 (no pain) to 10 (maximal pain) every 15 minutes for 3 hours.

Visual Analogue Score (VAS): The VAS is validated approach to pain measurement; it consists of a $10-\mathrm{cm}$ line with one end labeled "no pain" and the other end labeled "worst pain imaginable." The patient marks the line at the point that best describes the pain intensity (Jensen 1993)

F-Complications:

Nausea, vomiting, pruritus, shivering, desaturation or hypoxemia ( $\mathrm{SpO} 2$ $<90 \%$ ), respiratory depression (RR < 10), bradycardia and hypotension were also noticed and treated accordingly.

\section{Statistical analysis}

Data was analyzed using STATA intercooled version 12.1. Quantitative data was represented as mean, standard deviation, median and range. Data was analyzed using student t-test to compare means of two groups. When the data was not normally distributed Mann-Whitney test was used. Qualitative data was presented as number and percentage and compared using either Chi square test or fisher exact test. Graphs were produced by using Excel or STATA program. $\mathrm{P}$ value was considered significant if it was less than 0.05 . 


\section{Results}

There was No Statistically significant difference between Neostigmine group and Dexamethasone group as regard age or sex of patients

There was statistically significant difference between dexamethasone group and Neostigmine group group as regard Onset of sensory block as (P value Significant when $<0.05)$.As Dexamethasone group has faster onset of sensory block.; There was statistically significant difference between dexamethasone group and Neostigmine group sulfate group as regard as Duration of motor block as Dexamethasone group has longer duration of motor block; The duration of postoperative analgesia was prolonged in levobupivacaine-dexamethasone $(521.3 \pm 101.1 \mathrm{~min})$ and levo bupivacaine-neostigmine groups $(792.7 \pm 198.4 \mathrm{~min})$; There was no statistically significant difference between dexamethasone group and Neostigmine group as regard Onset of motor block; There was no statistically significant difference between dexamethasone group and Neostigmine group as regard Duration of Sensory Block

There was NO statistically significant difference between dexamethasone group and Neostigmine sulfate group as regard heart rate or oxygen saturation; As regard Systolic and Diastolic Blood pressure; Intraoperative there was Statistically Significant difference only in first 20 minutes then there was no Statistically Significant difference; Postoperative there was only Statistically Significant difference only in last 20 minutes.

As regard Complication that No Significant difference Between Two groups in rate of Complications; Hypotension and Bradycardia were most frequent complications in the two groups (graph.1)

\section{Discussion}

In our Study we Used 80 patients as follow:

Patients were divided into 2 equal groups:

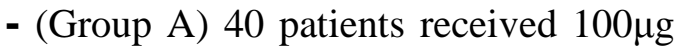
neostigmine in $0.5 \mathrm{ml}$ Normal saline added to $2.5 \mathrm{ml}$ volume of levobupivacaine

- (Group B) 40 patients received 4mg dexamethasone to $2.5 \mathrm{ml}$ volume of levobupivacaine.

The mechanism of analgesic effects of intrathecal dexamethasone is not fully cleared. Dexamethasone has direct action on the nerve membrane that causing local anesthetic effect which enhances the action of levobupivacaine and prolongs its duration and this effect may explain the analgesic effect of intrathecal dexamethasone (8).
Another possible mechanism is due to the action of dexamethasone on the spinal cord where dexamethasone may regulate nuclear factor- $\mathrm{kB}$ which is responsible for the development of pathological pain that can lead to inhibition of central sensitization and enhanced analgesic effect of CB (6).

The nuclear factor- $\mathrm{kB}$ is expressed in the nervous system and has a chief responsibility for pathogenesis of pathological pain (6).

As regards the analgesic effect of intrthecal dexamethasone administered in conjugation with local anesthetics solution, our results are on line with the results of Kim et al. who studied the effects of adding $0.1 \mathrm{mg} / \mathrm{kg}$ dexamethasone to ropivacaine $0.15 \%$ in 80 pediatric patients scheduled for ambulatory surgery, orchiopexy. Ropivacaine $0.15 \% \quad(1.5 \mathrm{ml} / \mathrm{kg})$ or 
ropivacaine $0.15 \% \quad(1.5 \mathrm{ml} / \mathrm{kg}) \quad$ with dexamethasone of $0.1 \mathrm{mg} / \mathrm{kg}$ were given to children for caudal epidural analgesia. They proved that co-administration of dexamethasone with ropivacaine significantly improved the analgesic quality. Choudhary et al. evaluated the analgesic effect of co-administration of dexamethasone $0.1 \mathrm{mg} / \mathrm{kg}$ to ropivacaine $0.2 \%$ for $\mathrm{CB}$ in 120 children underwent hernia repair. They concluded that the use of dexamethasone as an adjuvant to ropivacaine for $\mathrm{CB}$ produced longer post-operative pain relief period with lower pain score than those produced by ropivacaine. Several studies proved the analgesic effect of dexamethasone when added to local anesthetic solution (7).

The analgesic effect of intrathecal neostigmine is explained by inhibition of breakdown of the central neurotransmitter acetylcholine le ading to increased acetylcholine concentration in cerebrospinal fluid (7)

The analgesic effect is done via activation of spinal muscarinic M1 receptors and supraspinal muscarinic M1 and M2 and nicotinic cholinergic receptors (6).

As regards the analgesic effect of neostigmine $100 \mu \mathrm{g}$ when added to levobupivacaine, our results are in consistence with the results of Kumar et al. who studied the effects of adding ketamine $(0.5 \mathrm{mg} / \mathrm{kg})$,

midazolam $\quad(0.05 \mathrm{mg} / \mathrm{kg}), \quad$ and neostigmine $(2 \mu \mathrm{g} / \mathrm{kg})$ to $0.25 \%$ bupivacaine for $\mathrm{CB}$ in 80 pediatric patients underwent unilateral inguinal herniotomy. They concluded that coadministration of neostigmine or midazolam with bupivacaine for $\mathrm{CB}$ associated with prolonged postoperative analgesia.
Karaaslan et al. studied the effects of adding 2 or $4 \mu \mathrm{g} / \mathrm{kg}$ neostigmine to levobupivacaine $0.25 \% \quad(1 \mathrm{ml} / \mathrm{kg})$ for $\mathrm{CB}$ in 60 pediatric patients scheduled for genito-urinary surgery. They concluded that co-administration of neostigmine with levobupivacaine lengthened the postoperative pain free period without significant adverse events and $2 \mu \mathrm{g} / \mathrm{kg}$ neostigmine seemed to be the optimal dose. Mahajan et al. compared the effects of adding three doses of neostigmine $(2,3$ or $4 \mu \mathrm{g} / \mathrm{kg})$ to $0.25 \%$ bupivacaine $(0.5 \mathrm{ml} / \mathrm{kg})$ for $\mathrm{CB}$ in 80 children underwent surgery for hypospadias repair. They proved that the administration of neostigmine in doses of $2, \quad 3$ or $4 \mu \mathrm{g} / \mathrm{kg}$ with bupivacaine for $\mathrm{CB}$ resulted in better analgesic efficacy (approximately 16$17 \mathrm{~h}$ ) with reduction of postoperative analgesic requirement without significant side effects as compared to bupivacaine alone. The analgesic effect of intrathecal neostigmine when added to local anesthetic solution was documented in previous studies (6).

On the other hand, Memiş et al. concluded that administration of neostigmine $(1 \mu \mathrm{g} / \mathrm{kg})$ with bupivacaine $0.25 \%$ for CB did not improve postoperative analgesic duration in comparison to bupivacaine alone. The difference between their results and other investigators who documented the analgesic effectiveness of intrathecal neostigmine may be contributed to the low dose of neostigmine used by Memis et al. Bhardwaj et al. studied the effects of adding three doses of neostigmine (2, 3 or $4 \mu \mathrm{g} / \mathrm{kg}$ ) to bupivacaine $(0.75 \mathrm{ml} / \mathrm{kg})$ for $\mathrm{CB}$ in 120 children underwent urethroplasty surgery. They demonstrated that the co-administration of neostigmine with bupivacaine for $\mathrm{CB}$ 
did not lengthen the postoperative analgesia duration.

Animals and humans studies documented the safe utilization of neostigmine administrated either epidural or intrathecal (8).

\section{Conclusion}

In conclusion, the addition of Dexamethasone $4 \mathrm{mg}$ or Neostigmine $100 \mu \mathrm{g}$ to intrathecally injected Levobupivacaine improved the effect of Levobupivacaine and the postoperative analgesic effect and duration without serious significant changes in patients' hemodynamics and without serious complications when certain safety measures were taken.

\section{References}

1-Bani-Hashem N, Hassan-Nasab B, Pour EA, Maleh PA, Nabavi A, Jabbari A. Addition of intrathecal dexamethasone to bupivacaine for spinal anesthesia in orthopedic surgery. Saudi J Anaesth 2011; 5:382-386.

2- BROMAGE P.R.: An evaluation of bupivacaine in epidural analgesia for obstetrics. Can. Anesth. Soc. J., 16: 4656, 1969. Coated from Jaiswal P., Ranjan N., Tewari N., Agrawal R., Mathur S.K.: Comparative study of epidural midazolam and butorphanol as adjuvant with bupivacaine for labor analgesia: A double blind study. The Intenret Journal of Anesthesiology, Vol 14 Number 1, 2007.

3- BUVANENDRAN A., MCCARTHY R.J., KROIN J.S., LEONG W., PERRY P. and TUMAN K.J.: Intrathecal magnesium prolongs fentanyl analgesia:
A prospective, randomized, controlled trial. Anesth. Analg., 95: 661-6, 2002.

4-Gupta R, Bogra J, Verma R, Kohli M, Kushwaha JK, Kumar S. Dexmedetomidine as an intrathecal adjuvant for postoperative analgesia. Indian J Anaesth 2011; 55:347-351. *

5-Hisham Ahmed Fouad, Amal Mohammed Sabry Ahmed, Yasser Mohammed Mohammed Osman and Gamal Mohammed Taha Abouelmagd, Efficacy of primitive Dexamethasone added to Bupivacaine in ultrasound guided transverses abdominis plain block for POSTOPERATIVE analgesia after inguinal herniorrraphy. American Journal of Research Communication 2016

6-Movafegh A, Razazian M, Hajimaohamadi F, Meysamie A. Dexamethasone added to lidocaine prolongs axillary brachial plexus blockade. Anesth Analg 2006; 102:263267. I

7- Sapna Shashni, Abhijit S. Nair, T V S Gopal. Clinical effects of intrathecal midazolam versus intrathecal magnesium sulfate as adjuncts to hyperbaric bupivacaine: A comparative study. Indian journal of pain .Jan 2014.

8- -Lauretti GR , Azevedo VMS . Intravenous ketamine or fentanyl prologs postoperative analgesia after intrathecal neostigmine . Anesth Analg 1996 ; 83 :766 - 770 . 